\title{
Breaking time-reversal symmetry at the topological insulator surface by metal-organic coordination networks
}

\author{
M. M. Otrokov, ${ }^{1,2,3}$ E. V. Chulkov, ${ }^{1,4,3}$ and A. Arnau ${ }^{1,4}$ \\ ${ }^{1}$ Donostia International Physics Center (DIPC), 20018 San Sebastián/Donostia, Spain \\ ${ }^{2}$ Tomsk State University, 634050 Tomsk, Russia \\ ${ }^{3}$ Saint Petersburg State University, 198504 Saint Petersburg, Russia \\ ${ }^{4}$ Departamento de Física de Materiales UPV/EHU, Centro de Física de Materiales CFM-MPC and Centro Mixto CSIC-UPV/EHU, \\ 20080 San Sebastián/Donostia, Spain
}

(Received 5 June 2015; revised manuscript received 31 August 2015; published 12 October 2015)

\begin{abstract}
We propose a way to break the time-reversal symmetry at the surface of a three-dimensional topological insulator that combines features of both surface magnetic doping and magnetic proximity effect. Based on the possibility of organizing an ordered array of local magnetic moments by inserting them into a two-dimensional matrix of organic ligands, we study the magnetic coupling and electronic structure of such metal-organic coordination networks on a topological insulator surface from first principles. In this way, we find that both Co and $\mathrm{Cr}$ centers, linked by the tetracyanoethylenelike organic ligand, are coupled ferromagnetically and, depending on the distance to the topological insulator substrate, can yield a magnetic proximity effect. This latter leads to the Dirac point gap opening indicative of the time-reversal symmetry breaking.
\end{abstract}

DOI: 10.1103/PhysRevB.92.165309

PACS number(s): 73.20.At, 71.15.Mb, 71.20.Nr, 75.50.Xx

\section{INTRODUCTION}

Three-dimensional topological insulators (TIs), a novel class of materials in which an insulating bulk necessarily coexists with a metallic surface, have been attracting a great deal of attention for several years [1-4]. As a consequence of the strong spin-orbit coupling, the band gap in TIs gets inverted giving rise to a special type of surface states that have linear dispersion characteristic of Dirac fermions. The uniqueness of these Dirac states lies in that the electron's spin is locked perpendicular to its momentum, forming a surface state's spin structure which protects the electrons from the elastic backscattering on defects or due to disorder [5-9]. This property, provided by the time-reversal symmetry, leads to a nearly dissipationless current whereby TIs possess an enormous potential for applications in spintronics and quantum computing. To date, the research trend of TIs has developed into a standalone field giving rise to the subfields of Weyl [10] and threedimensional Dirac [11] semimetals, while similar physics has also been found in graphene-based systems [12,13].

Although it is time-reversal symmetry what topologically protects the electrons from backscattering, an interesting consequence arises upon its breaking. The latter can be realized by adding magnetic functionality to TI, i.e., either by surface [14-16] or bulk [17-22] doping with magnetic atoms or bringing the TI in contact with (an) a (anti)ferromagnetic [(A)FM] material [23-29]. In the presence of FM order with an out-of-plane magnetization the TI energy band structure becomes fully gapped due to the splitting of the Dirac point (DP). In such a case, the quantum anomalous Hall effect is expected to appear [30], which might establish a ground for the realization of the fault-tolerant electronic devices.

The strategy of bulk doping has already yielded fruits and quantum anomalous Hall effect was observed in $\mathrm{Cr}_{x}\left(\mathrm{Bi}_{1-y} \mathrm{Sb}_{y}\right)_{2-x} \mathrm{Te}_{3}[31,32]$ and $\mathrm{V}_{0.11}\left(\mathrm{Bi}_{0.29} \mathrm{Sb}_{0.71}\right)_{1.89} \mathrm{Te}_{3}$ [33] magnetic TI thin films, although at extremely low temperatures of 30 and $130 \mathrm{mK}$, respectively. The drawback of the low operational temperatures can in principle be overcome by bringing TI in contact with (an) a (A)FM compound. For example, the proximity of the AFM insulator $\mathrm{MnSe}$ to $\mathrm{Bi}_{2} \mathrm{Se}_{3}$ induces the gap of $\sim 8.5 \mathrm{meV}$ at the DP [26]. However, such an approach may lead to the appearance of undesirable trivial states [24,26,29] inside the TI bulk band gap which are harmful for the topological transport.

As far as the surface doping by the magnetic atoms is concerned, the issue of the DP gap opening proves to be controversial so far. On the one hand, $a b$ initio calculations performed for ordered arrays of $\mathrm{Co}$ [34] and $\mathrm{Cr}$ [35] adatoms at the $\mathrm{Bi}_{2} \mathrm{Se}_{3}$ surface predict FM ordering, out-of-plane magnetization, and, therefore, the DP splitting. This is in agreement with angle-resolved photoemission spectroscopy (ARPES) measurements of magnetically doped bismuth chalcogenide surfaces exhibiting a feature near the DP which resembles a gap [36,37]. On the other hand, magnetic measurements found no evidence of the long-range FM order in the systems of $\mathrm{Fe}$ and $\mathrm{Co}$ adatoms at the $\mathrm{Bi}_{2} \mathrm{Te}_{3}$ [38] and $\mathrm{Bi}_{2} \mathrm{Se}_{3}$ [39-41] surfaces (the coverage in the latter case was reaching 0.9 monolayer). Accordingly, several ARPES studies report the absence of the DP gap opening in such systems [40-45]. The suppression of the long-range FM order may stem from the nonuniform distribution of magnetic adsorbates on the TI surface, that tend to form clusters when their coverage is around 0.1 monolayer or higher $[39,40,44,46]$. This situation may be partly overcome by annealing, as it triggers the diffusion of the adsorbates inside the TI bulk, where they substitute Bi atoms [44,47,48]. It was predicted theoretically, that such an alloying favors the long-range FM order and out-of-plane magnetization that leads to the DP gap opening in $\mathrm{Bi}_{1.8} \mathrm{Mn}_{0.2} \mathrm{Te}_{3}[49,50]$. Nevertheless, a recent ARPES experiment [44] on $\mathrm{Fe}$ at $\mathrm{Bi}_{2} \mathrm{Se}_{3}$ doesn't support this result, but the amount of the deposited Fe was twice as small as that of $\mathrm{Mn}$ in $[49,50]$.

Here we propose a way to avoid the above described problems of the adatoms clustering and inhomogeneous distribution aiming at meeting the conditions for the timereversal symmetry breaking via the surface magnetic doping. 
Namely, to achieve the long-range FM order in the system of adatoms at the TI surface one has to organize their ordered arrays $[14,34,35]$ and prevent them from diffusion $[44,47]$ or intercalation inside the bulk or the van der Waals gaps [34,5154]. Lately, a type of system which may provide these conditions were successfully grown on metallic substrates-selfassembled metal-organic coordination networks (MOCNs) [55-64]. In such experiments, strong electron acceptors like tetracyanoethylene (TCNE, $\mathrm{C}_{6} \mathrm{H}_{4}$ ), tetracyanobenzene (TCNB, $\mathrm{C}_{10} \mathrm{H}_{2} \mathrm{~N}_{4}$ ), and tetracyanoquinodimethane (TCNQ, $\mathrm{C}_{12} \mathrm{H}_{4} \mathrm{~N}_{4}$ ) are the most frequently used molecules. Their networks represent themselves the two-dimensional hosts where the metallic centers carrying magnetic moment can reside. Therefore, one is able to organize an ordered array of magnetic adatoms thus suppressing their clustering. The latter is provided by the strong chemical bonding to the cyano groups of the organic ligands which prevents adatoms from diffusion on the surface or below it. Importantly, the local spins of the metallic centers don't quench upon contact with the substrate and the FM exchange interactions mediated by the organic ligands have been shown to exist in Fe- and Ni-based MOCNs deposited on the noble metal surfaces $[58,60,64]$.

Hitherto, the [organic molecules]/TIs interfaces were organized only in order to tune the transport properties of TI surfaces [65-68]. A well-ordered purely organic layer has also been reported to form on graphene [69]. However, in the recent work [70] the formation of the well-defined networks of Co and $\mathrm{Cu}$ phthalocyanine at the $\mathrm{Bi}_{2} \mathrm{Te}_{3}$ TI surface have been reported. Unfortunately, although one of the systems under consideration contained $\mathrm{Co}$ atoms, its magnetic properties were out of the work [70] scope.

Conceptually, the deposition of the MOCNs at TIs surfaces can be considered as a "hybrid method" to break the timereversal symmetry as it bears features of both surface magnetic doping and magnetic proximity effect simultaneously. The former because the magnetic atoms are located at the surface, the latter due to the presence of well-defined interface between magnetic and nonmagnetic systems. The important question to be answered in this context is whether this kind of system can produce the proximity effect which is strong enough to break the time-reversal symmetry at TI surface. Here we study the general characteristic features of the MOCN/TI systems as exemplified by a representative organic ligand (an artificial TCNE analog) linking together $\mathrm{Co}$ or $\mathrm{Cr}$ magnetic centers on the surface of the $\mathrm{Bi}_{2} \mathrm{Se}_{3}$-based heterostructure [71]. We explore possible adsorption geometries, magnetic properties and electronic structure of such MOCNs/TI. The consequences of an incommensurate growth of MOCN/TI are also discussed. We believe that the results obtained reflect general characteristic features of the TCNE-, TCNB-, and TCNQ-based MOCNs deposited on top of tetradymite-type TIs [8,72-74]. The main conclusion of this study is that the time-reversal symmetry can indeed be broken by the deposition of a MOCN on top of the TI surface.

\section{METHOD AND MODEL DESCRIPTION}

The calculations were carried out using the projector augmented-wave method [75] as implemented in the VASP code [76-78]. The generalized gradient approximation to the

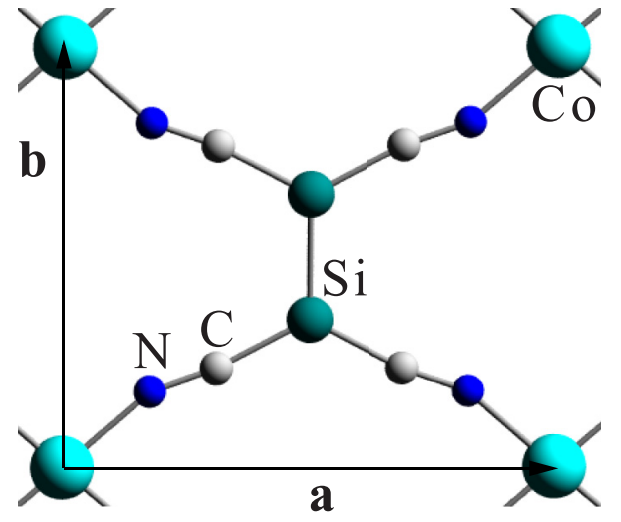

FIG. 1. (Color online) Unit cell of the unsupported Co-TC(Si)NE monolayer constrained to the parameters of the $\mathrm{Sb}_{2} \mathrm{Se}_{2} \mathrm{Te} / \mathrm{Bi}_{2} \mathrm{Se}_{3}$ heterostructure. We assume, for clarity, that $\mathbf{a}(\mathbf{b})$ is perpendicular (parallel) to the Si-Si bond.

exchange-correlation potential in the Perdew-Burke-Ernzerhof version [79] was employed. All calculations were performed using a model of repeating slabs separated by a vacuum gap of a minimum of $10 \AA$; the energy cutoff for the plane-wave expansion was set to $400 \mathrm{eV}$.

The MOCNs considered in this study consisted of Co or Cr metallic centers connected by modified TCNE molecules in which two central carbon atoms were replaced by two silicon atoms (Fig. 1). Below, this "synthetic" molecule will be referred to as TC(Si)NE. Such a replacement of the TCNE central carbon pair by that of silicon was made in order to achieve better lattice matching with the underlying TI, since the one provided by TCNE was rather large. The reasonable matching is crucial for our ability to study the system using a first-principles method. Otherwise one has to consider an incommensurate interface, modeling which will require a very large in-plane cell. The latter, in combination with a big thickness of the slab [at least six quintuple layers (QLs)] and spin-orbit coupling, needed for correct description of the TI surface band structure, makes the density functional theory study of MOCN/TI with an incommensurate interface practically impossible. We stress that $\mathrm{TC}(\mathrm{Si}) \mathrm{NE}$ is used as a representative organic ligand. Apart from TCNE, it bears common features with such other molecules as, e.g., TCNB and TCNQ. First, all these molecules are strong electron acceptors which is provided by the presence of four peripheral cyano groups. Second, they are planar and have rectangular shape. We have artificially designed TC(Si)NE using TCNE as a "parent" molecule since it is the smallest one among those mentioned. The small size of $\mathrm{TC}(\mathrm{Si}) \mathrm{NE}$ and its reasonable matching to $\mathrm{Bi}_{2} \mathrm{Se}_{3}$ allow us to choose relatively small in-plane cell- $(2 \times \sqrt{3}) a_{0}$, where $a_{0}$ is the $\mathrm{Bi}_{2} \mathrm{Se}_{3}$ in-plane lattice parameter. Such a MOCN/TI system ( $\sim 180$ atoms) is computationally affordable for the first-principles method.

In fact, the substrate was chosen to be not $\mathrm{Bi}_{2} \mathrm{Se}_{3}$, but the $\mathrm{Sb}_{2} \mathrm{Se}_{2} \mathrm{Te} / \mathrm{Bi}_{2} \mathrm{Se}_{3}$ (SST/BS) heterostructure [71], consisting of a single $\mathrm{Sb}_{2} \mathrm{Se}_{2} \mathrm{Te} \mathrm{QL}$ block on top of the $\mathrm{Bi}_{2} \mathrm{Se}_{3}$ substrate. This complex system had been preferred to the simple $\mathrm{Bi}_{2} \mathrm{Se}_{3}$ due to the fact that deposition of the $\mathrm{Co}(\mathrm{Cr})-\mathrm{TC}(\mathrm{Si}) \mathrm{NE}$ on top of $\mathrm{Bi}_{2} \mathrm{Se}_{3}$ leads to a significant potential bending as a 
result of which the DP falls into the continuum of the valence band states thus acquiring a resonance character. In contrast, SST/BS doesn't suffer from this drawback, since its DP is originally located close to the conduction band minimum [71]. As in the case of $\mathrm{Bi}_{2} \mathrm{Se}_{3}, \mathrm{SST} / \mathrm{BS}$ provides a reasonable lattice matching to both the $\mathrm{Co}-$ and $\mathrm{Cr}-\mathrm{TC}(\mathrm{Si}) \mathrm{NE}$ MOCNs.

All structural optimizations were performed within the scalar-relativistic approach using a conjugate-gradient algorithm and a force tolerance criterion for convergence of $0.05 \mathrm{eV} / \AA$. In the first step, the in-plane lattice parameters and the atomic coordinates of the free-standing MOCNs were optimized to determine their equilibrium structure. After that, the lattice parameters were constrained to those of SST/BS and the atomic coordinates were relaxed accordingly. The equilibrium adsorption geometry, i.e., the MOCN's $x y$ position with respect to the substrate and the distance to it, were then determined. For that purpose, only the SST QL block was used to model the substrate. In order to account for the van der Waals interactions we made use of DFT-D2 approach proposed by Grimme [80]. Once the adsorption geometry was established, the magnetic order was investigated by means of the scalar-relativistic total-energy calculations performed for the FM phase and three different AFM phases (horizontal and vertical stripes, as well as a checkerboard phase). This was done using $(2 \times 2)$ surface supercells with four metal atoms, that allow treating all these spin structures on equal footing. Finally, as soon as the FM order at $T=0$ is confirmed for the particular MOCN system, the surface electronic structure calculations of the MOCN-on-TI heterostructure with the spin-orbit coupling included by the second variation method [81] were performed. Symmetric slabs composed of $\mathrm{Co}(\mathrm{Cr})-\mathrm{TC}(\mathrm{Si}) \mathrm{NE} /\left[\mathrm{Sb}_{2} \mathrm{Se}_{2} \mathrm{Te}\right]_{1 \mathrm{QL}} /\left[\mathrm{Bi}_{2} \mathrm{Se}_{3}\right]_{6 \mathrm{QL}}$ in the $(2 \times$ $\sqrt{3}$ ) in-plane geometry were used. Formally, this can be considered as a $\mathrm{Co}(\mathrm{Cr})$ coverage of 0.25 monolayer. In all calculations (including those with the duplicated cells) a $\bar{\Gamma}$-centered $k$-point grid of $4 \times 5 \times 1$ was used to sample the two-dimensional Brillouin zone.

According to a recent study by Faraggi et al. [64], the electronic and magnetic structure of the $3 d$ transition metal atoms based MOCNs is very sensitive to the specific $3 d$ manyfold occupation that may give rise to delocalized or localized spin densities depending on the existence of hybridizations between $3 d$ states and molecular orbitals. Therefore, in the present work the $3 d$ states of $\mathrm{Co}$ or $\mathrm{Cr}$ were treated within the GGA $+U$ approach [82] and Dudarev's scheme [83]. The $U-J$ value of $4 \mathrm{eV}$ was used for both $\mathrm{Co}$ and $\mathrm{Cr}$ in all calculations.

\section{RESULTS AND DISCUSSION}

Table I reports the equilibrium lattice parameters of the unsupported $\mathrm{Co}$ - and $\mathrm{Cr}-\mathrm{TC}(\mathrm{Si}) \mathrm{NE}$ as well as their mismatches to those of the SST/BS substrate. It is seen that Co-TC(Si)NE's $b$ parameter matches quite well to that of SST/BS since $\Delta_{b}=0.85 \%$, while the $a$ parameter of the MOCN is $7.44 \%$ greater than the respective substrate parameter. The matching of the $\mathrm{Cr}-\mathrm{TC}(\mathrm{Si}) \mathrm{NE}$ is qualitatively the same but slightly worse quantitatively. The mismatch obtained can to some extent be accommodated with the bending of the MOCN units. Indeed, the conformational freedom of the organic acceptor increases upon the uptake of electrons and the cyano groups are then able
TABLE I. Equilibrium lattice parameters $a$ and $b$ (in $\AA$ ) of the free-standing Co- and Cr-TC(Si)NE, the local spin moments of the metallic centers (in $\mu_{\mathrm{B}}$ ), and the lattice parameters' mismatches $\Delta$ (in \%) with the SST/BS substrate. For SST/BS, $a=a_{0} \sqrt{3}=7.12 \AA$ and $b=2 a_{0}=8.22 \AA$, where $a_{0}=4.11 \AA$ is the $\mathrm{Bi}_{2} \mathrm{Se}_{3}$ parameter.

\begin{tabular}{lccccc}
\hline \hline & $a(\AA)$ & $b(\AA)$ & $\Delta_{a}(\%)$ & $\Delta_{b}(\%)$ & $m\left(\mu_{\mathrm{B}}\right)$ \\
\hline $\mathrm{Co}$ & 7.65 & 8.29 & 7.44 & 0.85 & 2.10 \\
$\mathrm{Cr}$ & 7.64 & 8.46 & 7.30 & 2.92 & 3.63 \\
\hline \hline
\end{tabular}

to bend $[63,84]$. According to the results of a recent theoretical study [64], there is a significant charge transfer (between 1 and $2 e^{-}$) from the $\mathrm{Ni}$ and $\mathrm{Mn}$ atoms to the TCNQ lowest unoccupied molecular orbital (LUMO). This could enable the bending of the cyano groups thus facilitating a complete or partial accommodation of the mismatch. Interestingly, both $\mathrm{Co}$ and $\mathrm{Cr}$ atoms feature significant local magnetic moments (Table I) and, therefore, their respective MOCNs are good candidates for the deposition on the TI substrate aiming at the opening of the DP, provided they order ferromagnetically and show out-of-plane magnetization.

The compression of the MOCNs to the lattice parameters of the substrate is expected to affect their structural properties. Indeed, we found that when constrained to the SST/BS lattice, both $\mathrm{Co}-$ and $\mathrm{Cr}-\mathrm{TC}(\mathrm{Si}) \mathrm{NE}$ change their internal atomic structure in such a way that $\mathrm{Si}-\mathrm{Si}-\mathrm{C}$ connection somewhat decreases its angle. As a result, the $a>b$ relation takes place (as opposite to the equilibrium case, see Table I), and the energy gain with respect to the constrained $a<b$ case is equal to 0.033 and $0.514 \mathrm{eV}$ per unit cell (31 atom) for $\mathrm{Co}-$ and $\mathrm{Cr}-\mathrm{TC}(\mathrm{Si}) \mathrm{NE}$, respectively. Since for $\mathrm{Co}-\mathrm{TC}(\mathrm{Si}) \mathrm{NE}$ this gain is marginal, the next step, i.e., determination of the adsorption geometry, was performed for both $a>b$ and $a<b$ cases. This was done for $\mathrm{Cr}-\mathrm{TC}(\mathrm{Si}) \mathrm{NE}$ as well.

To investigate the adsorption geometry, i.e., the $x y$ position of the MOCN with respect to the substrate and the adsorption height, we performed static total-energy calculations for 15 different distances between the substrate and MOCN. At that, we explored four different registries of the metallic center with respect to the substrate-top, bridge, fcc, and hcp hollows. For both MOCNs, the calculations were performed for the previously determined structures with $a$ both larger and smaller than $b$. Note that the MOCNs were kept planar during these calculations. It appeared that the $a>b$ case is again more favorable than the $a<b$ one. Indeed, the $E_{a<b}^{\min }-E_{a>b}^{\min }$ value, i.e., the energy difference of the two more favorable structures of these situations, amounted to $0.85 \mathrm{eV}$ $[0.83 \mathrm{eV}]$ for $\mathrm{Co}-\mathrm{TC}(\mathrm{Si}) \mathrm{NE}$ [Cr-TC(Si)NE]. Figure 2, showing the curves for the $a>b$ case, summarizes the results of these calculations. One can see that once placed above SST, both Coand $\mathrm{Cr}-\mathrm{TC}(\mathrm{Si}) \mathrm{NE}$ strongly prefer the top structure in which the metallic center resides right above one of the topmost layer Se atoms. The equilibrium adsorption heights for planar MOCNs amount to $\sim 2.85 \AA$ and $\sim 2.90 \AA$ for Co- and Cr-based systems, respectively. Since the metal centers are located in top positions, these values correspond to the Co-Se and $\mathrm{Cr}$-Se bond length, i.e., there is chemical bonding between metallic centers and Se atom. As far as the bridge, hcp, and $f c c$ configurations 


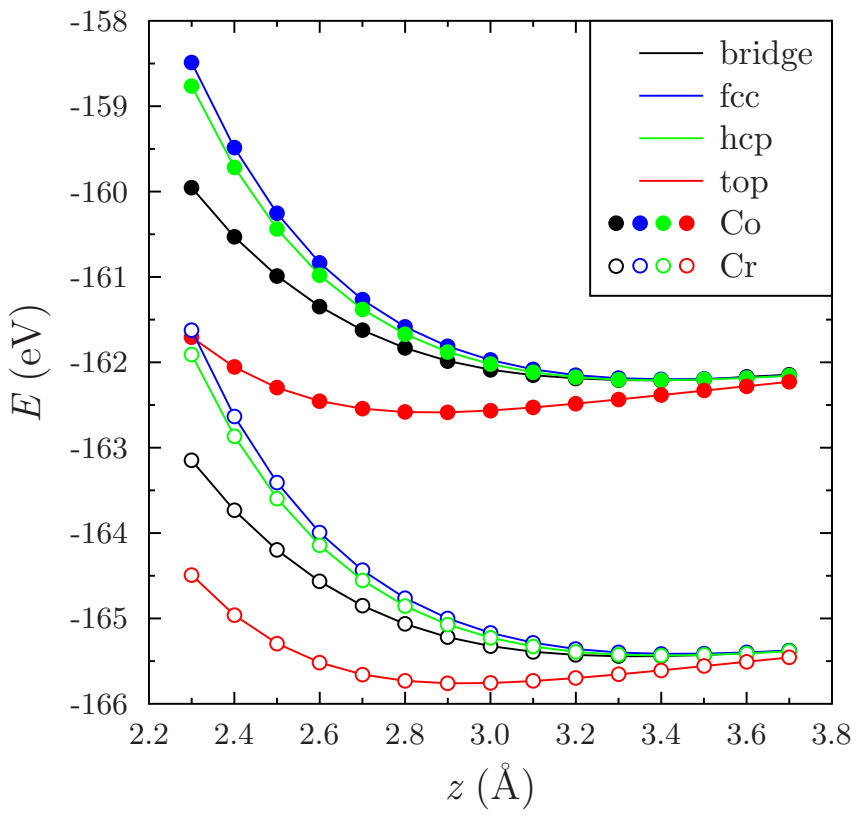

FIG. 2. (Color online) Total energy of the Co- (solid circles) and $\mathrm{Cr}-\mathrm{TC}(\mathrm{Si}) \mathrm{NE} / \mathrm{SST}$ (open circles) as a function of the adsorption distance for different MOCNs' registries with respect to the SST substrate (color lines). The $a>b$ case is shown.

are concerned, the MOCNs would appear to be physisorbed at roughly $3.4 \AA$ above SST.

We then found that in the described top geometry the metallic centers tend to get closer to the substrate. Therefore, they were allowed to relax downwards until the forces acting on them were less than $0.05 \mathrm{eV} / \AA$. As a result, the Co$\mathrm{Se}(\mathrm{Cr}-\mathrm{Se})$ bond length was reduced to $2.75 \AA$ ( $2.79 \AA)$. Further relaxations of the TC(Si)NE atoms revealed that it is extremely difficult to diminish the forces somewhat noticeably. Therefore, the organic ligands were kept fixed in both cases. However, such an incomplete relaxation is not expected to affect the results and conclusions of the present work by two reasons. First, the adsorption registry (top) is not expected to change since it is by far more favorable than the other adsorption geometries. Indeed, in the Co-MOCN (Cr-MOCN) case the chemisorbed top registry is $0.375 \mathrm{eV}(0.317 \mathrm{eV})$ more favorable than the bridge registry, which has the lowest energy among the three physisorbed cases-bridge, $f c c$, and $h c p$ ones. Second, the organic ligand atoms don't play a significant role in the magnetic proximity effect. Therefore, their displacements will not affect the magnetic proximity effect qualitatively. As far as the quantitative side is concerned, we recall that the purpose of our study is not to propose certain system, but rather explore and extract the general features of the FM MOCNs on top of TIs. Therefore we further study the MOCNs/TI in such a "metastable" geometry. Additionally, we treat the adsorption distance as a parameter, varying it in the physically meaningful range.

It is appropriate here to discuss in more detail the mismatch between the lattice constants of the free-standing MOCNs and the TI substrate. As is shown below, there are two possible scenarios when a MOCN and TI are brought in contact, namely, an incommensurate and commensurate growth of a
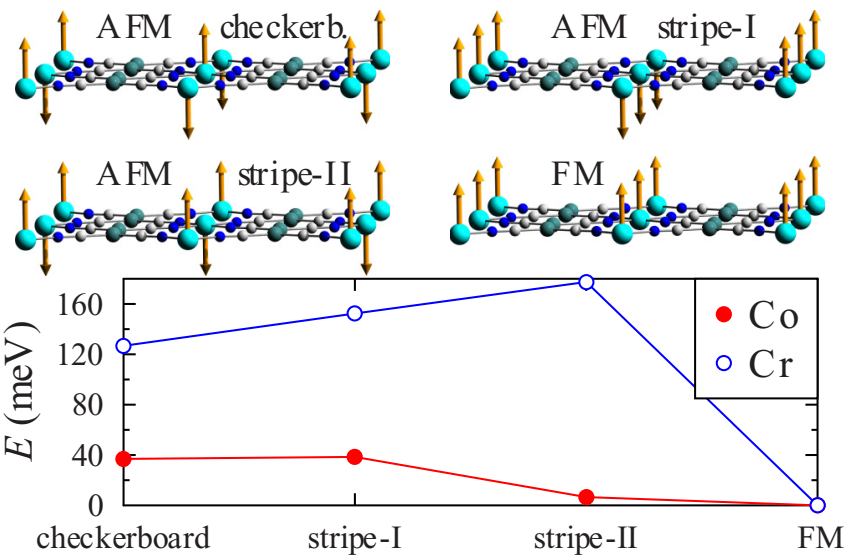

FIG. 3. (Color online) Total energies of the three AFM spin structures (shown above), the checkerboard, stripe-I, and stripe-II, with respect to the energy of the FM one. Energies are in meV per two local moments. The lines are guides to the eyes.

MOCN. The realization of these scenarios depends on how much energy gains the resultant system from the interface formation. Let us illustrate these two cases by the examples of $\mathrm{Co}-$ and $\mathrm{Cr}-\mathrm{TC}(\mathrm{Si}) \mathrm{NE}$. As it can be seen from Table I, the Co-TC(Si)NE MOCN matches SST/BS somewhat better than the $\mathrm{Cr}-\mathrm{TC}(\mathrm{Si}) \mathrm{NE}$ one. In the former, the top registry is so favorable (see above), that the energy gain due to the chemical bonding to the substrate $(0.375 \mathrm{eV})$ turns out to be comparable to the energy loss of $0.38 \mathrm{eV}$ due to the constraining of the free-standing Co-TC(Si)NE to the SST/BS parameters. Therefore, in principle, one cannot exclude the possibility of the commensurate interface formation in the case of particular MOCN and TI. Concerning the $\mathrm{Cr}-\mathrm{TC}(\mathrm{Si}) \mathrm{NE}$ case, the worse matching leads to the higher cost of the commensurability $(0.607 \mathrm{eV})$. Therefore, the energy gain of the chemisorbed top case of $0.317 \mathrm{eV}$ is far from being enough to make the commensurate growth favorable and formation of the incommensurate interface is expected in such cases.

Figure 3 shows the calculated energies of the different collinear magnetic structures for the above described bent geometries of Co- and Cr-TC(Si)NE/SST. The energy of the FM state is taken as zero in both cases. It is seen that both systems feature a FM ground state and that ferromagnetism proves to be energetically much more stable in the Cr-based MOCN. Thus, in the MOCN/TI systems, there exists an essential possibility to realize the magnetically ordered state: magnetic moments of the $\mathrm{Co}$ and $\mathrm{Cr}$ centers aren't quenched (2.08 and $3.35 \mu_{B}$, respectively) and there is an exchange interaction between them.

Next, we proceed with a detailed analysis of the calculated projected densities of states (PDOS) onto different $3 d$ metal atoms orbitals, $p$ orbitals of the cyano groups $\left(\mathrm{CN}^{-}\right)$, and $p_{z}$ orbital of the Se atom located directly below the metallic center. This analysis permits us to gain information about (i) the metallic/insulating character of the system and the localization of magnetic moments, (ii) the relevant in-plane and out-of-plane hybridizations between metal atoms and organic ligands, and (iii) the out-of-plane hybridizations 


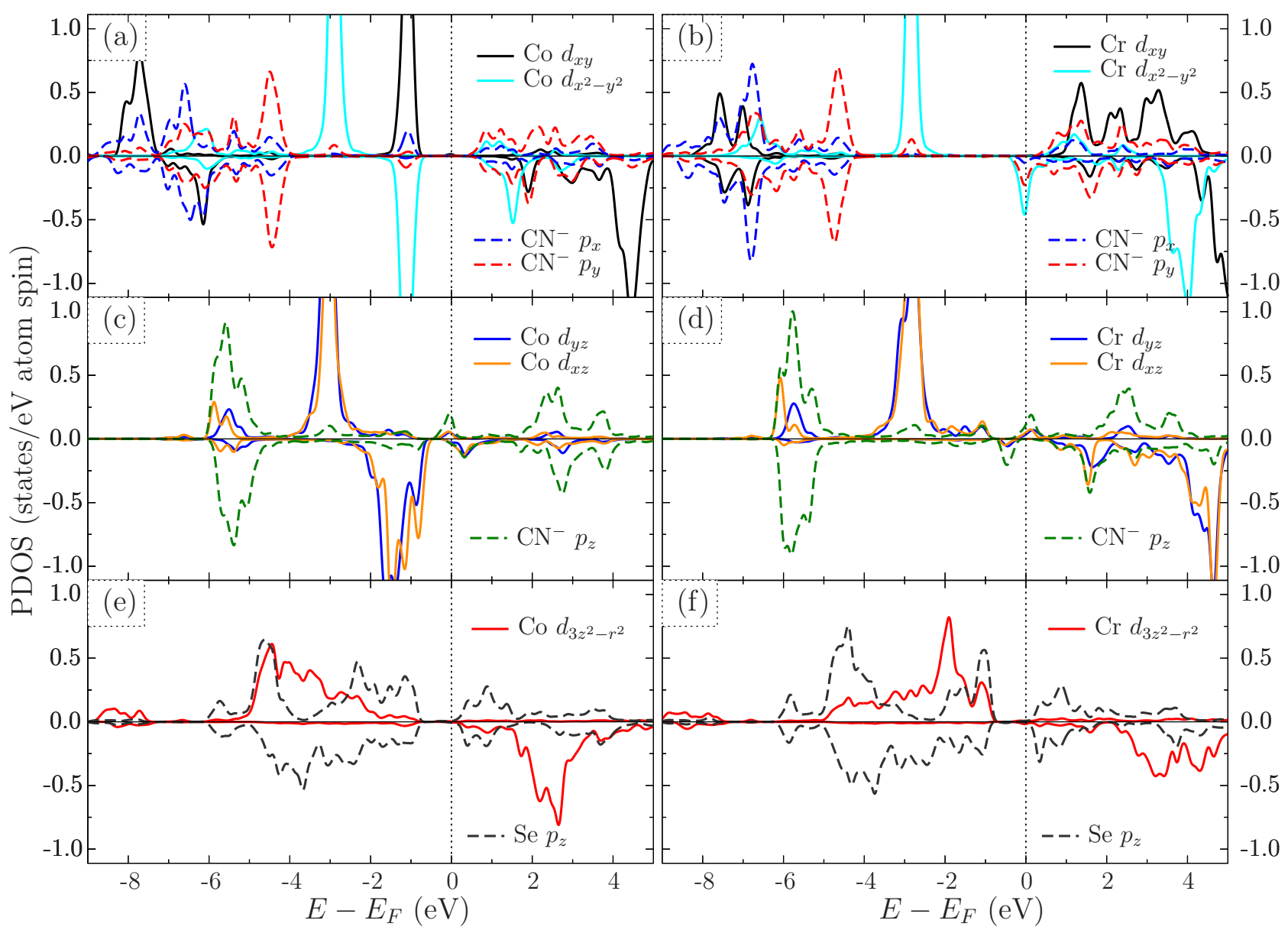

FIG. 4. (Color online) PDOS onto different atomic orbitals of Co- (a),(c),(e) and Cr-TC(Si)NE/SST (b),(d),(f) in FM state. Majority PDOS are plotted as positive values, while minority as negative ones. The vertical dotted line indicates the Fermi energy.

between metal atoms and the underlaying substrate. The corresponding PDOS are presented in Fig. 4.

First, we focus on the global information extracted from a simple examination of peak positions and its location with respect to the Fermi level. We see that both Co- and $\mathrm{Cr}-\mathrm{TC}(\mathrm{Si}) \mathrm{NE} / \mathrm{SST}$ are metallic since the Fermi level, $E_{\mathrm{F}}$, crosses the $d_{x z}$ and $d_{y z}$ projections of both spin subsystems in the former case [Fig. 4(c)] and the spin-down $d_{x^{2}-y^{2}}$ as well as spin-up $d_{x z}$ and $d_{y z}$ states in the latter case [Figs. 4(b) and $4(\mathrm{~d})$ ]. Concerning the $3 d$ metal atom orbital occupations that determine the corresponding magnetic moments, we observe that the majority spin states of Co are almost fully occupied, while the minority $d_{3 z^{2}-r^{2}}$ [Fig. 4(e)] and, to a large extent, $d_{x y}$ [Fig. 4(a)] are empty giving rise to a $S=1$ local moment $\left(2.08 \mu_{B}\right.$ as calculated within Wigner-Seitz sphere). In the $\mathrm{Cr}$ case there are, roughly, four fully occupied majority orbitals $\left[d_{x^{2}-y^{2}}, d_{x z}, d_{y z}\right.$, and $d_{3 z^{2}-r^{2}}$; see Figs. 4(b), 4(d), and 4(f)] and partially occupied minority $d_{x^{2}-y^{2}}$ orbital [Fig. 4(b)] which leads to a localized spin moment somewhat larger than $S=3 / 2\left[3.35 \mu_{B}\right.$; the contribution of the occupied spin-up $d_{x y}$ state cancels by that of its spin-down counterpart; see Fig. 4(b)]. Furthermore, in the Co- and the Cr-based systems, there are unpaired electrons in the $d_{x y}$ and $d_{x^{2}-y^{2}}$ orbitals [Figs. 4(a) and 4(b)], respectively, what leaves out-of-plane $(|m|=2)$ orbital angular moment components. Besides, the
Fermi energy is located much closer to $3 d$ states which have mainly $d_{x z}$ and $d_{y z}\left(d_{x^{2}-y^{2}}\right)$ character in Co-MOCN $(\mathrm{Cr}-$ MOCN), corresponding to out-of-plane angular momentum states with $|m|=1(m=+2)[85,86]$. Thus there is a certain tendency to an out-of-plane magnetization in both $\mathrm{Co}-$ and $\mathrm{Cr}-\mathrm{TC}(\mathrm{Si}) \mathrm{NE}$ which is crucial for the DP gap opening at TI surface [30] (we recall that the in-plane magnetization leads to the shift of the DP out of the $\bar{\Gamma}$ point; see, e.g., Ref. [50]).

Second, we explore the different hybridizations between the atomic orbitals based on the symmetry considerations of the orbitals involved. We find the following. (i) The in-plane orbitals $d_{x y}$ and $d_{x^{2}-y^{2}}$ in both $\mathrm{Co}$ and $\mathrm{Cr}$ cases hybridize with the $p_{x}$ and $p_{y}$ states of the $\mathrm{CN}^{-}$, both below and above the Fermi level [Figs. 4(a) and 4(b)]. Concentrating on the occupied states only, for Co-TC(Si)NE the orbital overlaps are manifested in the appearance of the hybrid $d_{x^{2}-y^{2}}-p_{y}$ peaks at -2.9 and $-1.1 \mathrm{eV}$ of the spin-up and spin-down channels, respectively [Fig. 4(a)], while for $\mathrm{Cr}-\mathrm{TC}(\mathrm{Si}) \mathrm{NE}$ they appear at $-2.83 \mathrm{eV}$ and $E_{\mathrm{F}}$ [Fig. 4(b)]. The hybridization with the $d_{x y}$ states appears mostly below $-6 \mathrm{eV}$, except for Co-TC(Si)NE, which features a hybrid spin-up $d_{x y}-p_{x}$ peak at $-1.1 \mathrm{eV}$. (ii) The $\mathrm{CN}^{-}-p_{z}$ states hybridize with $d_{x z}$ and $d_{y z}$ [Figs. 4(c) and $4(\mathrm{~d})]$. Near the Fermi level these hybrid states form a so-called LUMO. As a result of these Co- and Cr-TC(Si)NE hybridizations, the LUMO becomes partially occupied and 

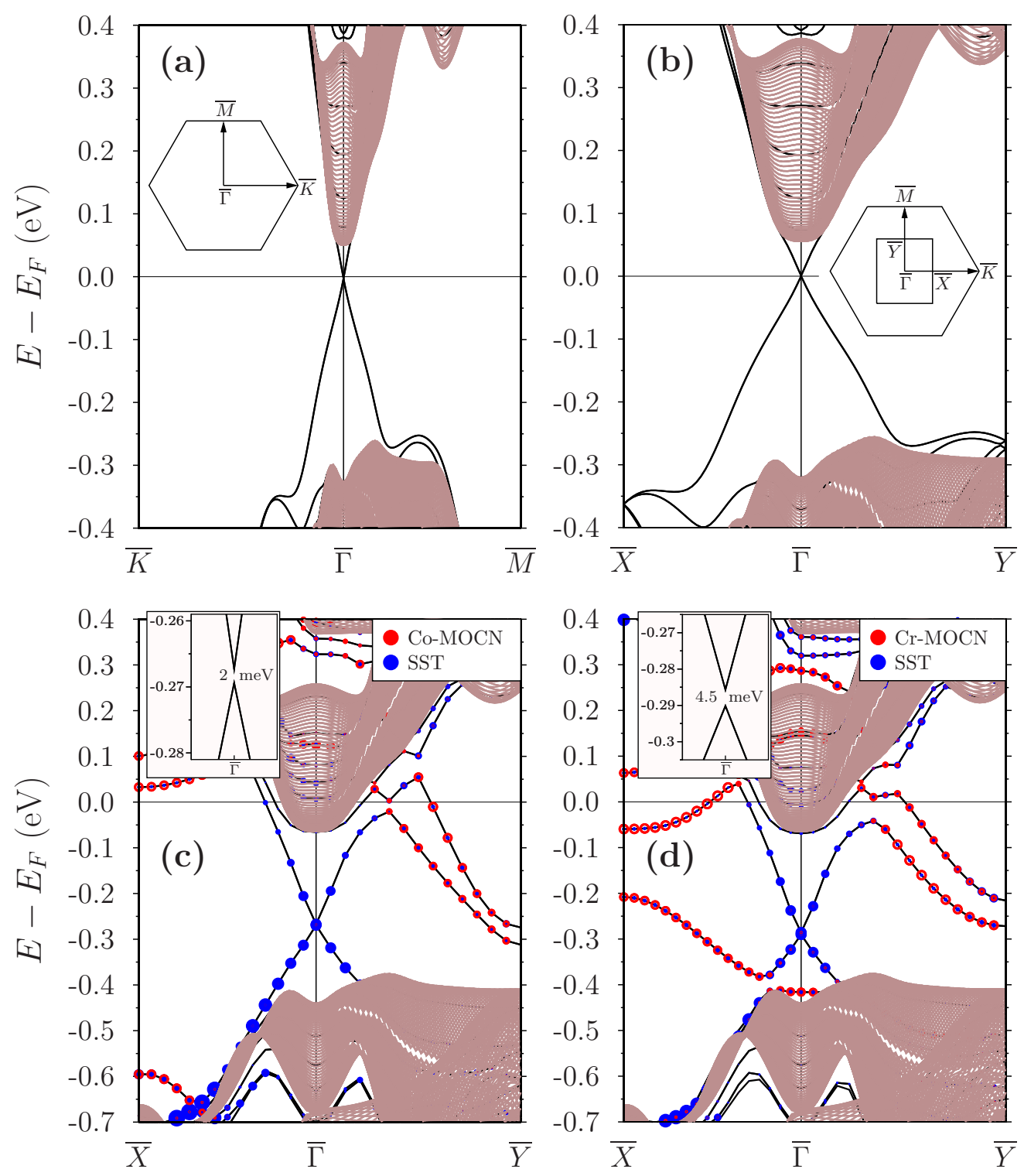

FIG. 5. (Color online) (a),(b) Surface band structure of SST/BS calculated in $(1 \times 1)$ and $(2 \times \sqrt{3})$ in-plane supercells. (c), (d) Surface band structure of the Co- and $\mathrm{Cr}-\mathrm{TC}(\mathrm{Si}) \mathrm{NE}$ networks placed on top of SST/BS. Purple-shaded areas correspond to the bulk band structure projected onto the surface Brillouin zone. The size of red and blue circles in panels (c),(d) reflects the weight of the state in the (Co,Cr)-MOCN and in the SST QL, respectively. The insets show the magnified views of the split DPs.

acquires spin polarization with the same (opposite) sign as that of the metallic $\mathrm{Co}(\mathrm{Cr})$ center, a prerequisite for FM coupling, as was recently shown in Ref. [64].

Finally, we consider the interaction of the metallic centers with the SST substrate. It is seen in Figs. 4(e) and 4(f) that the $d_{3 z^{2}-r^{2}}$ states hybridize with the $p_{z}$ states of the underlying Se atom. Although it is difficult to spot the common $p-d$ features, it is clear that the Se- $p_{z}$ states are strongly modified, as compared to those of the clean SST surface, where the spin-up and spin-down states are of identical shape. As for the Se- $p_{x}$ and $p_{y}$ states, they do not appear to be affected by the proximity of the MOCN (not shown). However, despite such a strong modification of the $p_{z}$ states, in the Co case there is almost no induced magnetization in the Se $p$ states: the calculated moment amounts to $\simeq-0.01 \mu_{B}$ (within the Wigner-Seitz sphere). Bringing Co-TC(Si)NE somewhat closer to the substrate, such that the Co-Se bond length is reduced to $2.5 \AA$ (instead of $2.75 \AA$ ) increases the mentioned induced moments roughly twice. Contrary to a Co center, the $\mathrm{Cr}$ one induces a notably larger moment of $-0.03 \mu_{B}$ in the $\mathrm{Se}$ $p$ states, and upon pushing $\mathrm{Cr}-\mathrm{TC}(\mathrm{Si}) \mathrm{NE}$ closer to the substrate it increases up to almost $-0.05 \mu_{B}$ (the Cr-Se bond length being equal to $2.5 \AA$ ). We note that the Se $p$-states magnetic moment induced by $\mathrm{Cr}-\mathrm{TC}(\mathrm{Si}) \mathrm{NE}$ is comparable to that induced at $\mathrm{Bi}$ atom, lying in the second atomic layer from the $\mathrm{Bi}_{2} \mathrm{Se}_{3} / \mathrm{MnSe}$ interface, by $\mathrm{Mn}$ atom lying at the interface [26]. Thus, at least in the Cr-TC(Si)NE/SST case, there is clear magnetic proximity effect, which is crucial for the gap opening at the three-dimensional TI surface [26].

Let us now discuss the electronic band structure of the systems when the Co- and $\mathrm{Cr}-\mathrm{TC}(\mathrm{Si}) \mathrm{NE}$ networks are placed on the complete SST/BS substrate. The calculations are 
performed with spin-orbit coupling included. The SST/BS surface band structure is shown in Fig. 5(a). Its characteristic feature is the DP lying close to the conduction band minimum [71] in contrast to the BS surface band structure showing the DP in the vicinity of the valence band maximum [74]. Figure 5(b) shows essentially the same SST/BS band structure but calculated in $(2 \times \sqrt{3})$ surface supercell, which is manifested in the folded surface bands near the $\bar{X}$ and $\bar{Y}$ points below the Fermi level. Then the band structures of the SST/BS with the MOCNs on top were calculated. It turned out that in the equilibrium geometry with metallic centers residing at 2.75$2.79 \AA$ above the substrate neither Co- nor Cr-based network produces the splitting of the DP larger than $1 \mathrm{meV}$ (not shown). To see whether the violation of time-reversal symmetry using the MOCN is ultimately feasible, the metal-organic overlayers were brought closer to the SST/BS substrate since it enhances the magnetic proximity effect, as it has already been discussed above. Figures 5(c) and 5(d) show the surface band structure of the Co- and $\mathrm{Cr}-\mathrm{TC}(\mathrm{Si}) \mathrm{NE}$ networks both located at $2.5 \AA$ above SST/BS. One can clearly see the splitting of the DP in both cases, with the gap sizes of 2 and $4.5 \mathrm{meV}$ in Co- and Cr-based systems, respectively. However, in neither case there is a global gap in the surface band structure, because the DP gaps are spanned by the trivial bands which appear inside the BS fundamental band gap at larger $\vec{k}$. These bands stem from the MOCN but also have a contribution from the substrate atoms due to the hybridization. It should also be noted that in the Cr case the DP splitting is of the same order of magnitude as that brought about by the BS proximity to the compound AFM semiconductor MnSe [26]. This is despite the fact that the effective concentration of the $\mathrm{Cr}$ atoms at the MOCN/SST interface is four times smaller than that of $\mathrm{Mn}$ atoms at the $\mathrm{Bi}_{2} \mathrm{Se}_{3} / \mathrm{MnSe}$ one.

The spectra shown in Fig. 5 are obtained under the assumption of commensurate growth of the MOCNs on top of SST/BS. Let us now briefly discuss possible consequences of the incommensurate growth illustrated by an example of our model $\mathrm{Cr}-\mathrm{TC}(\mathrm{Si}) \mathrm{NE} / \mathrm{SST} / \mathrm{BS}$ system. In this case, different $\mathrm{Cr}$ centers will have different atomic environment, i.e., the MOCN will not always be in the top registry, but there rather will be a continuous change of the registries. However, not all of them produce the magnetic proximity effect. Thus one can expect that in the case of the incommensurate growth the Dirac state will only be gapped in those regions, where this effect exists. In the case of Cr-TC(Si)NE/SST/BS it takes place when the MOCN is in the top registry or nearly. As for the regions where the $\mathrm{Cr}$ atoms fall into or close to the bridge, $f c c$, and $h c p$ sites, there was no magnetic proximity effect found and therefore no gap opening is expected. Due to the rectangular symmetry of the FM MOCNs under consideration they could create quasi-one-dimensional regions (stripes) of the gapped Dirac state at the TI surface. This is somewhat similar to the spatially dependent DP gap opening in the case of $\mathrm{Cr}_{x}\left(\mathrm{Bi}_{0.1} \mathrm{Sb}_{0.9}\right)_{2-x} \mathrm{Te}_{3}$ stemming from the inhomogeneous distribution of Cr dopants [87].

In Figs. 5(c) and 5(d) it can also be seen that the Dirac states are subject to a significant downward shift due to the potential bending produced by the deposition of the MOCN. Indeed, the shift of the split DP due to the deposition of Co-(Cr-)based MOCN amounts to $\sim 203$ (220) $\mathrm{meV}$, roughly the $2 / 3$ of the
BS bulk band gap. This means that wide band gap TIs [88] with the DP lying in the midgap or close to the conduction band minimum are suitable candidates for deposition of MOCNs aiming at a controlled modification of its electronic properties close to the Fermi level. The former could be obtained by a variation of bulk atomic composition [89,90], while the latter by constructing heterostructures $[71,91,92]$ like the one used in the present work.

Finally, it should be noted that the MOCN deposition results in a significant electron doping of the TI band structure (Fig. 5). Although the Fermi level position can be tuned into the bulk band gap by electrical gating, the use of $p$-doped TIs (e.g., $\mathrm{Sb}_{2} \mathrm{Te}_{3}$ [72]) as substrates for MOCNs deposition can help in decreasing the magnitude of the gate bias voltage required to access the Dirac state and the induced DP gap in particular.

\section{CONCLUSIONS}

The surface electronic structure of metal-organic coordination networks on top of a topological insulator surface has been studied using first-principles calculations. An analog of the tetracyanoethylene molecule with a central carbon pair substituted by a silicon one has been chosen as an organic ligand, representative of the flat, strong acceptor molecules characterized by the presence of the peripherally located cyano groups. Being used as a part of the metal-organic network with $3 d$ atoms acting as magnetic centers, it provides reasonable lattice matching to the topological insulators of the $\mathrm{Bi}_{2} \mathrm{Se}_{3}$ family, thus enabling an efficient $a b$ initio study of such a complex system. Thus we have been able to elicit general features, characteristic of a MOCN-on-TI, when a metal-organic coordination network is based on a flat, strong acceptor molecule (TCNE, TCNB, TCNQ, etc.), while a topological insulator belongs to the tetradymite family $\left(\mathrm{Sb}_{2-x} \mathrm{Bi}_{x} \mathrm{Te}_{3-y} \mathrm{Se}_{y}\right)$. The most important of these features are as follows. The deposition of a metal-organic coordination network on top of a topological insulator surface doesn't lead to a quenching of the $3 d$ atom local magnetic moments (at least in the Co and $\mathrm{Cr}$ cases) and there is a significant exchange interaction between them. Thus there exists a possibility for the realization of the magnetically ordered state in such systems. In the regions where the magnetic centers are chemically bound to the topological insulator surface, the magnetic proximity effect can be achieved. Finally, and most importantly, it has been shown that the deposition of the ferromagnetic metal-organic coordination networks can indeed provide all the conditions for time-reversal symmetry breaking at the topological insulator surface.

\section{ACKNOWLEDGMENTS}

We acknowledge the support by the Basque Departamento de Educacion, UPV/EHU (Grant No. IT-756-13), Spanish Ministerio de Economia y Competitividad (MINECO Grant No. FIS2013-48286-C2-2-P), and Tomsk State University Academic D.I. Mendeleev Fund Program in 2015 (Research Grant No. 8.1.05.2015). Partial support by the Saint Petersburg State University Project No. 11.50.202.2015 is also acknowledged. The calculations were performed in Donostia International Physics Center as well as on the SKIF-Cyberia supercomputer of Tomsk State University. 
[1] M. Z. Hasan and C. L. Kane, Rev. Mod. Phys. 82, 3045 (2010).

[2] T. Okuda and A. Kimura, J. Phys. Soc. Jpn. 82, 021002 (2013).

[3] Y. Ando, J. Phys. Soc. Jpn. 82, 102001 (2013).

[4] S. V. Eremeev, G. Landolt, T. V. Menshchikova, B. Slomski, Y. M. Koroteev, Z. S. Aliev, M. B. Babanly, J. Henk, A. Ernst, L. Patthey et al., Nat. Commun. 3, 635 (2012).

[5] P. Roushan, J. Seo, C. V. Parker, Y. S. Hor, D. Hsieh, D. Qian, A. Richardella, M. Z. Hasan, R. J. Cava, and A. Yazdani, Nature (London) 460, 1106 (2009).

[6] T. Hanaguri, K. Igarashi, M. Kawamura, H. Takagi, and T. Sasagawa, Phys. Rev. B 82, 081305 (2010).

[7] Z. Alpichshev, J. G. Analytis, J.-H. Chu, I. R. Fisher, Y. L. Chen, Z. X. Shen, A. Fang, and A. Kapitulnik, Phys. Rev. Lett. 104, 016401 (2010).

[8] P. Sessi, M. M. Otrokov, T. Bathon, M. G. Vergniory, S. S. Tsirkin, K. A. Kokh, O. E. Tereshchenko, E. V. Chulkov, and M. Bode, Phys. Rev. B 88, 161407 (2013).

[9] S. Roy, H. L. Meyerheim, A. Ernst, K. Mohseni, C. Tusche, M. G. Vergniory, T. V. Menshchikova, M. M. Otrokov, A. G. Ryabishchenkova, Z. S. Aliev et al., Phys. Rev. Lett. 113, 116802 (2014).

[10] A. A. Burkov and L. Balents, Phys. Rev. Lett. 107, 127205 (2011).

[11] S. M. Young, S. Zaheer, J. C. Y. Teo, C. L. Kane, E. J. Mele, and A. M. Rappe, Phys. Rev. Lett. 108, 140405 (2012).

[12] A. F. Young, J. D. Sanchez-Yamagishi, B. Hunt, S. H. Choi, K. Watanabe, T. Taniguchi, R. C. Ashoori, and P. Jarillo-Herrero, Nature (London) 505, 528 (2014).

[13] F. Calleja, H. Ochoa, M. Garnica, S. Barja, J. J. Navarro, A. Black, M. M. Otrokov, E. V. Chulkov, A. Arnau, A. L. V. de Parga et al., Nat. Phys. 11, 43 (2015).

[14] Q. Liu, C.-X. Liu, C. Xu, X.-L. Qi, and S.-C. Zhang, Phys. Rev. Lett. 102, 156603 (2009).

[15] D. A. Abanin and D. A. Pesin, Phys. Rev. Lett. 106, 136802 (2011).

[16] V. N. Men'shov, V. V. Tugushev, and E. V. Chulkov, JETP Lett. 94, 629 (2011).

[17] Y. L. Chen, J. H. Chu, J. G. Analytis, Z. K. Liu, K. Igarashi, H. H. Kuo, X. L. Qi, S. K. Mo, R. G. Moore, D. H. Lu et al., Science 329, 659 (2010).

[18] Y. S. Hor, P. Roushan, H. Beidenkopf, J. Seo, D. Qu, J. G. Checkelsky, L. A. Wray, D. Hsieh, Y. Xia, S.-Y. Xu et al., Phys. Rev. B 81, 195203 (2010).

[19] J. G. Checkelsky, J. Ye, Y. Onose, Y. Iwasa, and Y. Tokura, Nat. Phys. 8, 729 (2012).

[20] C.-Z. Chang, J. Zhang, M. Liu, Z. Zhang, X. Feng, K. Li, L.-L. Wang, X. Chen, X. Dai, Z. Fang et al., Adv. Mater. 25, 1065 (2013).

[21] X. F. Kou, W. J. Jiang, M. R. Lang, F. X. Xiu, L. He, Y. Wang, Y. Wang, X. X. Yu, A. V. Fedorov, P. Zhang, and K. L. Wang, J. Appl. Phys. 112, 063912 (2012).

[22] M. G. Vergniory, M. M. Otrokov, D. Thonig, M. Hoffmann, I. V. Maznichenko, M. Geilhufe, X. Zubizarreta, S. Ostanin, A. Marmodoro, J. Henk et al., Phys. Rev. B 89, 165202 (2014).

[23] P. Wei, F. Katmis, B. A. Assaf, H. Steinberg, P. Jarillo-Herrero, D. Heiman, and J. S. Moodera, Phys. Rev. Lett. 110, 186807 (2013).

[24] W. Luo and X.-L. Qi, Phys. Rev. B 87, 085431 (2013).
[25] Q. I. Yang, M. Dolev, L. Zhang, J. Zhao, A. D. Fried, E. Schemm, M. Liu, A. Palevski, A. F. Marshall, S. H. Risbud et al., Phys. Rev. B 88, 081407 (2013).

[26] S. V. Eremeev, V. N. Men'shov, V. V. Tugushev, P. M. Echenique, and E. V. Chulkov, Phys. Rev. B 88, 144430 (2013).

[27] V. N. Men'shov, V. V. Tugushev, S. V. Eremeev, P. M. Echenique, and E. V. Chulkov, Phys. Rev. B 88, 224401 (2013).

[28] M. Lang, M. Montazeri, M. C. Onbasli, X. Kou, Y. Fan, P. Upadhyaya, K. Yao, F. Liu, Y. Jiang, W. Jiang et al., Nano Lett. 14, 3459 (2014).

[29] S. Eremeev, V. Men'shov, V. Tugushev, and E. Chulkov, J. Magn. Magn. Mater. 383, 30 (2015)

[30] K. He, Y. Wang, and Q.-K. Xue, Natl. Sci. Rev. 1, 38 (2014).

[31] C.-Z. Chang, J. Zhang, X. Feng, J. Shen, Z. Zhang, M. Guo, K. Li, Y. Ou, P. Wei, L.-L. Wang et al., Science 340, 167 (2013).

[32] J. G. Checkelsky, R. Yoshimi, A. Tsukazaki, K. S. Takahashi, Y. Kozuka, J. Falson, M. Kawasaki, and Y. Tokura, Nat. Phys. 10, 731 (2014).

[33] C.-Z. Chang, W. Zhao, D. Y. Kim, H. Zhang, B. A. Assaf, D. Heiman, S.-C. Zhang, C. Liu, M. H. Chan, and J. S. Moodera, Nat. Mater. 14, 473 (2015).

[34] T. M. Schmidt, R. H. Miwa, and A. Fazzio, Phys. Rev. B 84, 245418 (2011).

[35] L. Chotorlishvili, A. Ernst, V. K. Dugaev, A. Komnik, M. G. Vergniory, E. V. Chulkov, and J. Berakdar, Phys. Rev. B 89, 075103 (2014).

[36] L. A. Wray, S.-Y. Xu, Y. Xia, D. Hsieh, A. V. Fedorov, Y. S. Hor, R. J. Cava, A. Bansil, H. Lin, and M. Z. Hasan, Nat. Phys. 7, 32 (2011).

[37] S.-Y. Xu, M. Neupane, C. Liu, D. Zhang, A. Richardella, L. A. Wray, N. Alidoust, M. Leandersson, T. Balasubramanian, J. Sanchez-Barriga et al., Nat. Phys. 8, 616 (2012).

[38] L. R. Shelford, T. Hesjedal, L. Collins-McIntyre, S. S. Dhesi, F. Maccherozzi, and G. van der Laan, Phys. Rev. B 86, 081304 (2012).

[39] J. Honolka, A. A. Khajetoorians, V. Sessi, T. O. Wehling, S. Stepanow, J.-L. Mi, B. B. Iversen, T. Schlenk, J. Wiebe, N. B. Brookes et al., Phys. Rev. Lett. 108, 256811 (2012).

[40] M. Ye, S. V. Eremeev, K. Kuroda, E. E. Krasovskii, E. V. Chulkov, Y. Takeda, Y. Saitoh, K. Okamoto, S. Y. Zhu, K. Miyamoto et al., Phys. Rev. B 85, 205317 (2012).

[41] M. Ye, K. Kuroda, Y. Takeda, Y. Saitoh, K. Okamoto, S.-Y. Zhu, K. Shirai, K. Miyamoto, M. Arita, M. Nakatake et al., J. Phys.: Condens. Matter 25, 232201 (2013).

[42] M. R. Scholz, J. Sánchez-Barriga, D. Marchenko, A. Varykhalov, A. Volykhov, L. V. Yashina, and O. Rader, Phys. Rev. Lett. 108, 256810 (2012).

[43] T. Valla, Z.-H. Pan, D. Gardner, Y. S. Lee, and S. Chu, Phys. Rev. Lett. 108, 117601 (2012).

[44] T. Schlenk, M. Bianchi, M. Koleini, A. Eich, O. Pietzsch, T. O. Wehling, T. Frauenheim, A. Balatsky, J.-L. Mi, B. B. Iversen et al., Phys. Rev. Lett. 110, 126804 (2013).

[45] E. Wang, P. Tang, G. Wan, A. V. Fedorov, I. Miotkowski, Y. P. Chen, W. Duan, and S. Zhou, Nano Lett. 15, 2031 (2015).

[46] T. Eelbo, M. Sikora, G. Bihlmayer, M. Dobrzański, A. Kozłowski, I. Miotkowski, and R. Wiesendanger, New J. Phys. 15, 113026 (2013).

[47] D. West, Y. Y. Sun, S. B. Zhang, T. Zhang, X. Ma, P. Cheng, Y. Y. Zhang, X. Chen, J. F. Jia, and Q. K. Xue, Phys. Rev. B 85, 081305 (2012). 
[48] A. Polyakov, H. L. Meyerheim, E. D. Crozier, R. A. Gordon, K. Mohseni, S. Roy, A. Ernst, M. G. Vergniory, X. Zubizarreta, M. M. Otrokov et al., Phys. Rev. B 92, 045423 (2015).

[49] J. Henk, A. Ernst, S. V. Eremeev, E. V. Chulkov, I. V. Maznichenko, and I. Mertig, Phys. Rev. Lett. 108, 206801 (2012).

[50] J. Henk, M. Flieger, I. V. Maznichenko, I. Mertig, A. Ernst, S. V. Eremeev, and E. V. Chulkov, Phys. Rev. Lett. 109, 076801 (2012).

[51] Y.-L. Wang, Y. Xu, Y.-P. Jiang, J.-W. Liu, C.-Z. Chang, M. Chen, Z. Li, C.-L. Song, L.-L. Wang, K. He et al., Phys. Rev. B 84, 075335 (2011).

[52] M. M. Otrokov, S. D. Borisova, V. Chis, M. G. Vergniory, S. V. Eremeev, V. M. Kuznetsov, and E. V. Chulkov, JETP Lett. 96, 714 (2013)

[53] M. A. Gosalvez, M. M. Otrokov, N. Ferrando, A. G. Ryabishchenkova, A. Ayuela, P. M. Echenique, and E. V. Chulkov, arXiv:1402.5920.

[54] A. G. Ryabishchenkova, M. M. Otrokov, V. M. Kuznetsov, and E. V. Chulkov, Zh. Eksp. Teor. Fiz. 148, 535 (2015).

[55] D. Wegner, R. Yamachika, Y. Wang, V. W. Brar, B. M. Bartlett, J. R. Long, and M. F. Crommie, Nano Lett. 8, 131 (2008).

[56] S. Bedwani, D. Wegner, M. F. Crommie, and A. Rochefort, Phys. Rev. Lett. 101, 216105 (2008).

[57] M. N. Faraggi, N. Jiang, N. Gonzalez-Lakunza, A. Langner, S. Stepanow, K. Kern, and A. Arnau, J. Phys. Chem. C 116, 24558 (2012).

[58] T. R. Umbach, M. Bernien, C. F. Hermanns, A. Krüger, V. Sessi, I. Fernandez-Torrente, P. Stoll, J. I. Pascual, K. J. Franke, and W. Kuch, Phys. Rev. Lett. 109, 267207 (2012).

[59] N. Abdurakhmanova, A. Floris, T.-C. Tseng, A. Comisso, S. Stepanow, A. De Vita, and K. Kern, Nat. Commun. 3, 940 (2012).

[60] N. Abdurakhmanova, T.-C. Tseng, A. Langner, C. S. Kley, V. Sessi, S. Stepanow, and K. Kern, Phys. Rev. Lett. 110, 027202 (2013).

[61] L. Giovanelli, A. Savoyant, M. Abel, F. Maccherozzi, Y. Ksari, M. Koudia, R. Hayn, F. Choueikani, E. Otero, P. Ohresser et al., J. Phys. Chem. C 118, 11738 (2014).

[62] F. Bebensee, K. Svane, C. Bombis, F. Masini, S. Klyatskaya, F. Besenbacher, M. Ruben, B. Hammer, and T. R. Linderoth, Ang. Chem. Int. Ed. 53, 12955 (2014).

[63] J. Rodríguez-Fernández, K. Lauwaet, M. A. Herranz, N. Martín, J. M. Gallego, R. Miranda, and R. Otero, J. Chem. Phys. 142, 101930 (2015).

[64] M. N. Faraggi, V. N. Golovach, S. Stepanow, T.-C. Tseng, N. Abdurakhmanova, C. S. Kley, A. Langner, V. Sessi, K. Kern, and A. Arnau, J. Phys. Chem. C 119, 547 (2015).

[65] D. Kim, S. Cho, N. P. Butch, P. Syers, K. Kirshenbaum, S. Adam, J. Paglione, and M. S. Fuhrer, Nat. Phys. 8, 459 (2012).

[66] D. Kim, Q. Li, P. Syers, N. P. Butch, J. Paglione, S. D. Sarma, and M. S. Fuhrer, Phys. Rev. Lett. 109, 166801 (2012).
[67] Y. Tanabe, K. K. Huynh, R. Nouchi, S. Heguri, G. Mu, J. Xu, H. Shimotani, and K. Tanigaki, J. Phys. Chem. C 118, 3533 (2014).

[68] J. Wang, A. S. Hewitt, R. Kumar, J. Boltersdorf, T. Guan, F. Hunte, P. A. Maggard, J. E. Brom, J. M. Redwing, and D. B. Dougherty, J. Phys. Chem. C 118, 14860 (2014).

[69] M. Garnica, D. Stradi, S. Barja, F. Calleja, C. Diaz, M. Alcami, N. Martin, A. L. Vazquez de Parga, F. Martin, and R. Miranda, Nat. Phys. 9, 368 (2013).

[70] T. Bathon, P. Sessi, K. A. Kokh, O. E. Tereshchenko, and M. Bode, Nano Lett. 15, 2442 (2015).

[71] T. V. Menshchikova, M. M. Otrokov, S. S. Tsirkin, D. A Samorokov, V. V. Bebneva, A. Ernst, V. M. Kuznetsov, and E. V. Chulkov, Nano Lett. 13, 6064 (2013).

[72] C. Seibel, H. Maaß, M. Ohtaka, S. Fiedler, C. Jünger, C.-H Min, H. Bentmann, K. Sakamoto, and F. Reinert, Phys. Rev. B 86, 161105 (2012).

[73] M. G. Vergniory, T. V. Menshchikova, S. V. Eremeev, and E. V. Chulkov, JETP Lett. 95, 213 (2012).

[74] S. Roy, H. L. Meyerheim, K. Mohseni, A. Ernst, M. M. Otrokov, M. G. Vergniory, G. Mussler, J. Kampmeier, D. Grützmacher, C. Tusche et al., Phys. Rev. B 90, 155456 (2014).

[75] P. E. Blöchl, Phys. Rev. B 50, 17953 (1994).

[76] G. Kresse and J. Furthmüller, Phys. Rev. B 54, 11169 (1996).

[77] G. Kresse and D. Joubert, Phys. Rev. B 59, 1758 (1999).

[78] G. Kresse and J. Hafner, Phys. Rev. B 47, 558 (1993).

[79] J. P. Perdew, K. Burke, and M. Ernzerhof, Phys. Rev. Lett. 77, 3865 (1996).

[80] S. Grimme, J. Comput. Chem. 27, 1787 (2006).

[81] D. D. Koelling and B. N. Harmon, J. Phys. C: Solid State Phys. 10, 3107 (1977).

[82] V. I. Anisimov, J. Zaanen, and O. K. Andersen, Phys. Rev. B 44, 943 (1991)

[83] S. L. Dudarev, G. A. Botton, S. Y. Savrasov, C. J. Humphreys, and A. P. Sutton, Phys. Rev. B 57, 1505 (1998).

[84] T.-C. Tseng, C. Urban, Y. Wang, R. Otero, S. L. Tait, M. Alcamí, D. Écija, M. Trelka, J. M. Gallego, N. Lin et al., Nat. Chem. 2, 374 (2010).

[85] P. Bruno, Phys. Rev. B 39, 865 (1989).

[86] G. H. O. Daalderop, P. J. Kelly, and M. F. H. Schuurmans, Phys. Rev. B 50, 9989 (1994).

[87] I. Lee, C. K. Kim, J. Lee, S. J. L. Billinge, R. Zhong, J. A Schneeloch, T. Liu, T. Valla, J. M. Tranquada, G. Gu et al., Proc. Natl. Acad. Sci. U.S.A. 112, 1316 (2015).

[88] I. V. Silkin, T. V. Menshchikova, M. M. Otrokov, S. V. Eremeev, Y. M. Koroteev, M. G. Vergniory, V. M. Kuznetsov, and E. V. Chulkov, JETP Lett. 96, 322 (2012).

[89] J. Zhang, C.-Z. Chang, Z. Zhang et al., Nat. Commun. 2, 574 (2011).

[90] T. Arakane, T. Sato, S. Souma et al., Nat. Commun. 3, 636 (2012).

[91] V. N. Men'shov, V. V. Tugushev, T. V. Menshchikova, S. V. Eremeev, P. M. Echenique, and E. V. Chulkov, J. Phys.: Condens. Matter 26, 485003 (2014).

[92] R. Shokri, H. L. Meyerheim, S. Roy, K. Mohseni, A. Ernst, M. M. Otrokov, E. V. Chulkov, and J. Kirschner, Phys. Rev. B 91, 205430 (2015). 